\title{
Caracterização morfológica de acessos de batata-doce do banco de germoplasma da UFVJM, Diamantina
}

\author{
Irã P Neiva ${ }^{1}$; Valter C de Andrade Júnior ${ }^{1}$; Daniel José S Viana ${ }^{1}$; José A Figueiredo ${ }^{1}$; Carlos V Mendonça \\ Filho $^{1}$; Rafael A da Costa Parrella ${ }^{2}$; João B Santos ${ }^{3}$ \\ 1UFVJM, C. Postal 38 39100-000 Diamantina-MG; iraagronomia@yahoo.com.br; valterjr@ufvjm.edu.br; djsviana@yahoo.com.br; \\ altairfig@yahoo.com.br; cvictor@jknet.com.br; ${ }^{2}$ Embrapa Milho e Sorgo, C. Postal 151, 35701-970, Sete Lagoas-MG; rafaelparrellla@ \\ bol.com.br; ${ }^{3}$ UFLA, C. Postal 37,37200-000 Lavras-MG; jbsantos@ufla.br
}

\begin{abstract}
RESUMO
O trabalho teve como objetivo caracterizar morfologicamente os acessos de batata-doce do banco de germoplasma da Universidade Federal dos Vales do Jequitinhonha e Mucuri (UFVJM). O experimento foi realizado no Setor de Olericultura da UFVJM, período de fevereiro a novembro de 2006, utilizando-se o delineamento em blocos casualizados (DBC), contendo 65 tratamentos e 3 repetições, totalizando 195 parcelas de $4,5 \mathrm{~m}^{2}$ cada. As avaliações da parte vegetativa foram feitas três meses após o plantio e a colheita de raízes foi realizada nove meses após o plantio das ramas. Observou-se pelo dendrograma de dissimilaridade morfológica, que os acessos da coleção do banco de germoplasma da UFVJM apresentaram ampla variabilidade morfológica. Os descritores referentes à parte aérea se mostraram significativos com diferença entre clones, pelo teste $\mathrm{Scott}-\mathrm{Knott}(\mathrm{p}=0,05)$, diferentemente daqueles referentes à raiz.
\end{abstract}

Palavras-chave: Ipomoea batatas, recursos genéticos, qualidade de raízes.

\begin{abstract}
Morphologic characterization of sweet potato accesses from the germplasm bank of the Universidade Federal dos Vales do Jequitinhonha e Mucuri, Brazil

Sweet potato accesses from the germplasm bank of the Universidade Federal dos Vales do Jequitinhonha e Mucuri, Diamantina, Minas Gerais state, Brazil were characterized. The experiment was carried out between February and November 2006, using a randomized blocks design, with 65 treatments and three replications, totaling 195 plots. The evaluation of the vegetative part was carried out three months after planting and the roots were harvested nine months after planting. Based on the dendrogram of morphologic differences we observed a great morphologic variability on the accesses. The characteristics of the vegetative part showed significant differences among the clones, by Scott Knot test at 5\% probability, differently from those of the roots.
\end{abstract}

Keywords: Ipomoea batatas, genetic resources, roots quality.

(Recebido para publicação em 30 de março de 2010; aceito em 20 de setembro de 2011) (Received on March 30, 2010; accepted on September 20, 2011)

\begin{abstract}
$\mathrm{A}^{\mathrm{b}}$ batata-doce (Ipomoea batatas (L) Lam.) é cultivada, praticamente, em toda a área do globo terrestre, onde tem usos múltiplos, tanto na alimentação humana como animal e também como matéria prima para a indústria. É uma cultura típica de áreas geográficas de baixa fertilidade, sendo considerada como uma cultura de subsistência, com amplas possibilidades de utilização (Brito, 2003). Possui cultivo bem disseminado em todas as regiões brasileiras, apresenta certa relevância econômica e é uma hortaliça de ampla aceitação popular, sendo cultivada na maioria das vezes por pequenos produtores rurais em sistemas agrícolas com reduzida utilização de insumos (Souza, 2000).

Em todo o mundo, a área plantada com batata-doce em 2006 foi de 8.661.288 ha, com um rendimento de 14,7 t ha-1 (FAO, 2010). A China se
\end{abstract}

destaca como o maior produtor mundial, com mais de 4,7 milhões de hectares cultivados com batata-doce, com produtividade média de 21,3 $\mathrm{t} \mathrm{ha}^{-1}$ de raízes. O continente africano se destaca como o segundo grande produtor, porém com baixa produtividade média de raízes $(4,4$ $\mathrm{t} \mathrm{ha}^{-1}$ ). O Brasil, em 2008, foi o décimo quinto produtor mundial de batata-doce, colhendo aproximadamente 518.000 toneladas de raízes, em uma área plantada superior a 48.000 ha, com produtividade média de $11.219 \mathrm{~kg} \mathrm{ha}^{-1}$ (IBGE, 2009). O Rio Grande do Sul é o estado maior produtor de batata-doce, com uma produção de 158.629 toneladas, que representa aproximadamente $31,0 \%$ da produção nacional. Em 2008, a área colhida com batata-doce no Rio Grande do Sul foi de 12.793,00 ha, com um rendimento médio de $12,59 \mathrm{t} \mathrm{ha}^{-1}$, enquanto que em Minas Gerais a área plantada e colhida foi de 1.169,00 ha, com um rendimento de 14,26 t ha-1 (IBGE, 2010). Apesar da importância alimentar que esta cultura representa, ainda carece de informações nos mais diversos campos da pesquisa, quando comparada com outras espécies de importância para o homem (Daros \& Amaral Júnior, 2000).

Apesar da alta variabilidade genética existente, as mudanças nos hábitos de consumo e a escassez de pesquisas com a cultura têm contribuído para a perda de genótipos importantes, sendo de extrema importância à manutenção de acessos de batata-doce em bancos de germoplasma, e a posterior avaliação em diferentes regiões de cultivo (Andrade Júnior et al., 2009; Viana, 2009; Figueiredo, 2010).

Poucas informações são disponíveis acerca de genótipos mais adaptados às condições particulares dos produtores de 
determinada região, devido à escassez de pesquisas. No entanto, há considerável diversidade genômica de batata-doce, oriunda da segregação sexuada e assexuada de introduções de plantas de outras localidades (Oliveira et al., 2000). Diante disso, é importante conhecer a divergência genética existente, identificando materiais genéticos muito próximos ou duplicados (Scapim et al., 1999; Oliveira et al., 2000).

A ocorrência de duplicatas não identificadas em bancos de germoplasma encarece e dificulta a manutenção adequada dos acessos, gerando problemas relacionados à organização e ao acesso de usuários potenciais ao recurso genético (Frankel \& Bennet, 1970; Strauss et al., 1989; Beuselinck \& Steiner, 1992).

A caracterização morfológica consiste em fornecer uma identidade para cada entrada por meio do conhecimento de uma série de dados que permita estudar a variabilidade genética de cada amostra (Ramos \& Queiroz, 1999), a fim de excluir a possibilidade de duplicatas e verificar a influência da morfologia no grupamento entre eles através de um dendrograma. O trabalho teve como objetivo caracterizar morfologicamente os acessos de batata-doce (Ipomoea batatas (L) Lam.) do banco de germoplasma da Universidade Federal dos Vales do Jequitinhonha e Mucuri (UFVJM).

\section{MATERIAL E MÉTODOS}

O experimento foi conduzido no Setor de Olericultura, localizado no campus JK da Universidade Federal dos Vales do Jequitinhonha e Mucuri (UFVJM), rodovia BR 367, no município de Diamantina-MG. O solo da área experimental foi classificado como Neossolo Quartizarênico Órtico Típico (Embrapa, 2007), e a análise química do mesmo, apresentou $\mathrm{pH}$ em água de $6,2 \%, \mathrm{P}$ de 144,5 ( $\left.\mathrm{mg} \mathrm{dm}^{-3}\right), 76 \mathrm{mg} \mathrm{dm}^{-3}$ de K, Ca de 2,0 ( $\left.\mathrm{cmol}_{\mathrm{c}} \mathrm{dm}^{-3}\right), \mathrm{Mg}$ de 1,3 $\left(\mathrm{cmol}_{\mathrm{c}} \mathrm{dm}^{-3}\right)$, Al de 0,1 ( $\left.\mathrm{cmol}_{\mathrm{c}} \mathrm{dm}^{-3}\right)$, $\mathrm{H}+\mathrm{Al}$ de $1,5\left(\mathrm{cmol}_{\mathrm{c}} \mathrm{dm}^{-3}\right), \mathrm{SB}$ de 3,5 $\left(\mathrm{cmol}_{\mathrm{c}} \mathrm{dm}^{-3}\right), \mathrm{t}$ de 3,6 ( $\left.\mathrm{cmol} \mathrm{dm}^{-3}\right), \mathrm{T}$ de

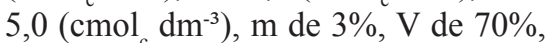
Matéria Orgânica 1,4 $\left(\mathrm{g} \mathrm{kg}^{-1}\right)$, areia de 810 , silte de $80\left(\mathrm{~g} \mathrm{dm}^{-3}\right)$, e argila de 110 $\left(\mathrm{g} \mathrm{dm}^{-3}\right)$.

O delineamento experimental utili- zado foi em blocos casualizados, contendo 65 tratamentos (Cambraia, Marmel, Batata Mandioca, Palmas, Arruba, Licuri, Espanhola, Tomba Carro, Coração Magoado, BD-01, BD-02, BD-03, BD-04, BD-05, BD-06, BD-07, BD-08, BD-10, BD-11, BD-13, BD-14, BD-15, BD-16, BD-17, BD-20, BD-21, BD-22, BD-23, BD-24, BD-25, BD-25 TO, BD-26, BD-28, BD-29, BD-30, BD-31 TO, BD-32, BD-33, BD-34, BD-36, BD-38, BD-39, BD-42, BD-43, BD-44, BD-45, BD-46, BD-47, BD-48, BD-49, BD-50, BD-51, BD-52, BD-53, BD-54, BD-56, BD-58, BD-65, BD-66, BD-67, BD-113 TO), materiais coletados em diversas regiões do Brasil, inclusive no Vale do Jequitinhonha, e as cultivares comerciais Brazlândia Roxa, Brazlândia Rosada, Coquinho e Princesa e 3 repetições, com um total de 195 parcelas de $4,5 \mathrm{~m}^{2}$ cada. $\mathrm{O}$ espaçamento utilizado foi de 1,0 m entre linhas (camalhão) e $30 \mathrm{~cm}$ entre plantas. As adubações de plantio, na dosagem de $20 \mathrm{~kg} \mathrm{ha}^{-1} \mathrm{de} \mathrm{N}$, $90 \mathrm{~kg} \mathrm{ha}^{-1}$ de $\mathrm{P}_{2} 0_{5}$ e $90 \mathrm{~kg} \mathrm{ha}^{-1}$ de $\mathrm{K}_{2} \mathrm{O}$, foram incorporadas nas leiras de cultivo, e a adubação de cobertura foi realizada aos 30 dias após o plantio das ramas utilizando $30 \mathrm{~kg} \mathrm{ha}^{-1}$ de $\mathrm{N}$ (Filgueira, 2008). Na fase inicial de crescimento das ramas foi realizada irrigação por aspersão, conforme recomendação de Bernardo et al. (2008). Os demais tratos culturais utilizados durante a condução do experimento foram realizados de acordo com as recomendações para a cultura da batata-doce (Filgueira, 2008).

O plantio foi realizado nos dias 08 , 09 e 10 de fevereiro de 2006, utilizando-se ramas selecionadas e padronizadas com 8 nós, enterrando-se de 3 a 4 nós. Foram realizados replantios das ramas após 15, 18, 20 e 22 dias. As avaliações da parte vegetativa foram feitas três meses após o plantio. A colheita foi realizada, cerca de nove meses após o plantio das ramas. Após a colheita, procedeu-se a avaliação das características de qualidade das raízes. As partes vegetativas foram avaliadas, conforme preconizado por Huamán (1996): a) tamanho da folha (TF) [pequena $(<8 \mathrm{~cm})$, média $(8-15 \mathrm{~cm})$, grande $(16-25 \mathrm{~cm})$ ou muito grande $(>25 \mathrm{~cm})]$, com notas $3 ; 5$. 7 , ou 9 , respectivamente; b) perfil geral da folha (PG) (arredondada, reniforme, cordada, triangular, lanceolada, lobulada ou quase dividida), com notas $1 ; 2 ; 3$; $4 ; 5 ; 6$ ou 7 , respectivamente; c) tipo de lóbulo da folha (TL) (ausência de lóbulos, lóbulos muito superficiais, lóbulos superficiais, moderados, profundos ou muito profundos), com notas $0 ; 1 ; 3$; $5 ; 7$ ou 9 , respectivamente; d) número de lóbulos da folha (NL), obtido pela contagem dos lóbulos de cada folha, descartando-se os basais; e) cor da folha madura (FM) (amarelo-verde, verde, verde com bordas roxas, verde cinzento, verde com nervuras roxas na face, ligeiramente roxa, predominantemente roxa, verde na face superior e roxo na inferior ou roxo em ambas as superfícies), com notas $1 ; 2 ; 3 ; 4 ; 5 ; 6 ; 7 ; 8$ ou 9 , respectivamente; $f$ ) cor da folha imatura (FI) (amarelo-verde, verde, verde com bordas roxas, verde cinzento, verde com nervuras roxas na face, ligeiramente roxa, predominantemente roxa, verde na face superior e roxo na inferior ou roxo em ambas as superfícies), com notas 1 ; $2 ; 3 ; 4 ; 5 ; 6 ; 7 ; 8$ ou 9 , respectivamente; g) comprimento do pecíolo (COM) [muito curto $(<10 \mathrm{~cm})$, curto $(10-20 \mathrm{~cm})$, intermediário (21-30 cm), longo (31-40 $\mathrm{cm})$ ou muito longo $(>40 \mathrm{~cm})]$, com notas $1 ; 3 ; 5 ; 7$ ou 9 , respectivamente; h) pigmentação das nervuras inferiores (PNI) (amarelo, verde, mancha roxa na base da nervura principal, mancha roxa em várias nervuras ou nervura principal parcialmente roxa, nervura principal predominantemente ou totalmente roxa, todas nervuras parcialmente roxas, todas as nervuras predominantemente ou totalmente roxas, toda face inferior ou nervuras totalmente roxas), com notas 1 ; $2 ; 3 ; 4 ; 5 ; 6 ; 7 ; 8$ ou 9 , respectivamente.

Para as raízes foram avaliadas, segundo Huamán (1996): a) cor secundária das ramas (CS) (ausente, base verde, ápice verde, nós verdes, base rosada, ápice rosado, nós rosados ou outros), com notas $0 ; 1 ; 2 ; 3 ; 4 ; 5 ; 6$ ou 7 , respectivamente; b) pubescência do ápice das ramas (PAR) (ausência de pilosidade, pilosidade rala, moderada, densa ou muito densa), com notas $0 ; 3$; $5 ; 7$ ou 9 , respectivamente; c) formato da raiz (FOR) [redondo (perfil quase circular com uma proporção de largura e comprimento de 1:1), redondo elíptico (perfil ligeiramente circular com bordos 
agudos e uma proporção de largura e comprimento de 2:1), elíptico (perfil com a largura máxima igual à distância de ambos os extremos e uma proporção de largura e comprimento não superior a 3:1), ovado (perfil semelhante a um ovo), obovado (perfil inverso ao ovado), oblongo (perfil quase retangular com os lados paralelos), largo oblongo (perfil oblongo com uma proporção de largura e comprimento superior a 3:1), largo elíptico (perfil elíptico com uma proporção de largura e comprimento superior a 4:1) ou largo irregular ou curvado, com notas $1 ; 2 ; 3 ; 4 ; 5 ; 6 ; 7$; 8 ou 9 , respectivamente; d) defeitos na superfície da raiz (DS) (ausente, periderme com pele de crocodilo, veias proeminentes, constrições horizontais superficiais, constrições horizontais profundas, fendas longitudinais superficiais, fendas longitudinais profundas, constrições e rachaduras profundas ou outros), com notas $0 ; 1 ; 2 ; 3 ; 4 ; 5 ; 6 ; 7$ ou 8 , respectivamente; e) resistência a insetos do solo (RI) (raízes livres de danos causados por insetos, com aspecto comercial desejável; raízes com poucos danos, mas com presença de algumas galerias e furos nas raízes; raízes com danos verificados sem muito esforço visual (presença de galeria e furos nas raízes em maior intensidade), com aspecto comercial prejudicado; raízes com muitos danos, praticamente imprestáveis para comercialização (presença de muitas galerias, furos e início de apodrecimento); raízes totalmente imprestáveis para fins comerciais (repletas de galerias, furos e apodrecimento mais avançado)), com notas $1 ; 2 ; 3 ; 4$ ou 5 , respectivamente.

Foram feitas análises estatísticas utilizando o programa SISVAR (Ferreira, 2003) e o teste agrupamento de média Scott-Knott (1974) e obtenção da matriz de correlação fenotípica pelo Programa GENES (Cruz, 1997).

Para as análises de diversidade, os dados tomados quantitativamente foram padronizados conforme Domingues et al. (1999) e foi utilizada a distância Euclidiana e para o agrupamento foi obtido o dendrograma para os pares ordenados pela média aritmética não ponderada (UPGMA) utilizando-se o programa "Statistica" para "Windows" (StatSoft, Inc., 2000).

\section{RESULTADOS E DISCUSSÃO}

Houve diferença significativa a $1 \%$ pelo teste $\mathrm{F}$ entre os clones para todas as características avaliadas, exceto para resistência a insetos do solo, formato de raiz e defeitos na superfície da raiz. Verifica-se variação quanto ao coeficiente de variação $(\mathrm{CV})$, onde nos caracteres com CV acima de $30 \%$, mostra a grande dificuldade na avaliação dos mesmos (Tabela 1). Daros et al. (2002), em experimento similar, observaram maior variação para os caracteres de parte aérea em relação aos de raiz. Já Azevedo (1995) avaliando clones de batata-doce quanto ao formato de raízes, constatou diferença significativa entre os clones, com notas de formato variando de 1,90 a 3,67. Cardoso et al. (2005) não encontraram diferença significativa entre os clones avaliados para resistência a insetos de solo.

De acordo com a matriz de correlação fenotípica para as variáveis avaliadas (Tabela 2), o tipo de lóbulo da folha (TL) apresentou forte correlação com o perfil geral da folha (PG), sendo igual a 0,87, e também com o número de lóbulo $(\mathrm{NL})$, igual a 0,86 , sendo altamente significativo. Já o perfil geral da folha (PG), se mostrou altamente correlacionado com número de lóbulo (NL), igual a 0,83 , mostrando que são caracteres bem similares, ou seja, que há possibilidades de apenas um deles ser avaliado, e neste caso, há indícios que o PG possa ter sido avaliado com maior precisão.

Com base nas médias dos dados morfológicos obtidos a partir dos clones, obteve-se a matriz de distâncias euclidianas, a partir da qual se gerou o dendrograma (Figura 1). Observa-se que houve a formação de dois grupos distintos com subdivisões. O primeiro grupo reúne os seguintes clones: BD28, BD-39, BD-66, BD-13, Palmas, Coquinho, BD-49, Cambraia, Marmel, BD-33, BD-5, BD-14, BD-04, Coração Magoado, BD-44, BD-43, Tomba Carro, Licuri, BD-20, BD-26 e BD-02. Os clones reunidos no segundo grupo foram: BD-29, BD-25, BD-46, BD-38, BD-31TO, BD-45, BD-24, BD-67, BD22, Brazlândia Rosada, BD-65, BD-48, BD-113TO, BD-36, BD-21, BD-30,

Tabela 1. Resumo das análises de variância para as características avaliadas (summary of the analysis of variance for the characteristics evaluated). Diamantina, UFVJM, 2008.

\begin{tabular}{|c|c|c|c|c|c|c|c|c|c|c|c|c|c|c|}
\hline \multirow{2}{*}{ FV } & \multirow{2}{*}{ GL } & \multicolumn{13}{|c|}{ QM } \\
\hline & & TF & PG & NL & FM & FI & PNI & CS & TL & COM & PAR & RI & FOR & DSR \\
\hline Bloco & 2 & 3,30 & 0,17 & 0,84 & 0,04 & 1,49 & 1,66 & 0,29 & 1,49 & 1,72 & 35,75 & 0,11 & 7,45 & 0,06 \\
\hline $\begin{array}{l}\text { Trata- } \\
\text { mentos }\end{array}$ & 64 & $0,29 * *$ & $2,28 * *$ & $5,94 * *$ & $4,64 * *$ & $2,24 * *$ & $4,90 * *$ & $17,91 * *$ & $15,70 * *$ & $0,53 * * 1$ & $11,96 * *$ & 0,34 & 1,04 & 0,52 \\
\hline Erro & 128 & 0,10 & 0,19 & 0,33 & 0,94 & 0,62 & 0,45 & 1,70 & 0,89 & 0,17 & 2,25 & 0,26 & 0,88 & 0,47 \\
\hline $\mathrm{CV}$ & & 6,94 & 9,55 & 17,52 & 37,89 & 32,39 & 16,77 & 36,63 & 27,22 & 20,98 & 53,89 & 25,73 & 13,49 & 46,13 \\
\hline Média & & 4,68 & 4,63 & 3,29 & 2,56 & 2,44 & 4,01 & 3,56 & 3,47 & 1,94 & 2,79 & 1,97 & 6,96 & 1,49 \\
\hline
\end{tabular}

**Significativo a $1 \%$ pelo teste $\mathrm{F} ; \mathrm{TF}=$ leaf size (tamanho da folha); $\mathrm{PG}=$ general profile of the leaf (perfil geral da folha); $\mathrm{NL}=$ number of lobes (número de lóbulos); FM= mature leaf color (cor da folha madura); FI= immature leaf color (cor da folha imatura); $\mathrm{CS}=$ secondary color of the branches (cor secundária das ramas); $\mathrm{TL}=$ type of leaf lobes (tipo de lóbulo da folha); COM= petiole length (comprimento do pecíolo); $\mathrm{PAR}=$ pubescence of the stem apex (pubescência do ápice das ramas); $\mathrm{RI}=$ insect resistance to soil insects (resistência a insetos do solo); $\mathrm{FOR}=$ root format (formato da raiz); $\mathrm{DSR}=$ defects on the surface of the root (defeitos na superfície da raiz). 
Tabela 2. Matriz de correlação fenotípica para as variáveis avaliadas (phenotypic correlation matrix for the variables evaluated). Diamantina, UFVJM, 2008.

\begin{tabular}{|c|c|c|c|c|c|c|c|c|c|c|c|c|c|}
\hline & TF & PG & NL & FM & FI & PNI & $\mathrm{CS}$ & TL & COM & PAR & $\mathbf{R I}$ & FOR & DSR \\
\hline TF & 1 & $-0,12$ & $-0,09$ & 0,14 & $-0,03$ & $-0,01$ & $-0,16$ & $-0,14$ & $0,54 * *$ & 0,06 & $-0,01$ & $-0,14$ & 0,07 \\
\hline PG & & 1 & $0,83 * *$ & $0,39 * *$ & 0,14 & 0,10 & $0,41 * *$ & $0,87 * *$ & $-0,03$ & $-0,08$ & $-0,12$ & 0,00 & 0,29 \\
\hline NL & & & 1 & $0,38^{* *}$ & 0,09 & 0,11 & $0,37 * *$ & $0,86^{* *}$ & 0,01 & $-0,10$ & $-0,14$ & 0,11 & 0,34 \\
\hline FM & & & & 1 & 0,32 & 0,12 & 0,30 & $0,50^{* *}$ & $-0,02$ & 0,06 & $-0,15$ & 0,07 & 0,00 \\
\hline FI & & & & & 1 & 0,12 & $-0,13$ & 0,15 & $-0,20$ & 0,02 & 0,01 & 0,08 & 0,01 \\
\hline PNI & & & & & & 1 & 0,28 & 0,26 & 0,05 & 0,16 & 0,00 & $-0,06$ & $-0,21$ \\
\hline CS & & & & & & & 1 & $0,53 * *$ & $-0,14$ & 0,22 & $-0,16$ & $-0,02$ & $-0,11$ \\
\hline TL & & & & & & & & 1 & $-0,06$ & $-0,06$ & $-0,18$ & 0,16 & 0,27 \\
\hline COM & & & & & & & & & 1 & 0,10 & $-0,03$ & 0,00 & 0,04 \\
\hline PAR & & & & & & & & & & 1 & $-0,07$ & 0,04 & $-0,14$ \\
\hline RI & & & & & & & & & & & 1 & $-0,23$ & $-0,19$ \\
\hline FOR & & & & & & & & & & & & 1 & 0,34 \\
\hline DSR & & & & & & & & & & & & & 1 \\
\hline
\end{tabular}

**Significativo a $1 \%$ pelo teste $\mathrm{T}$.

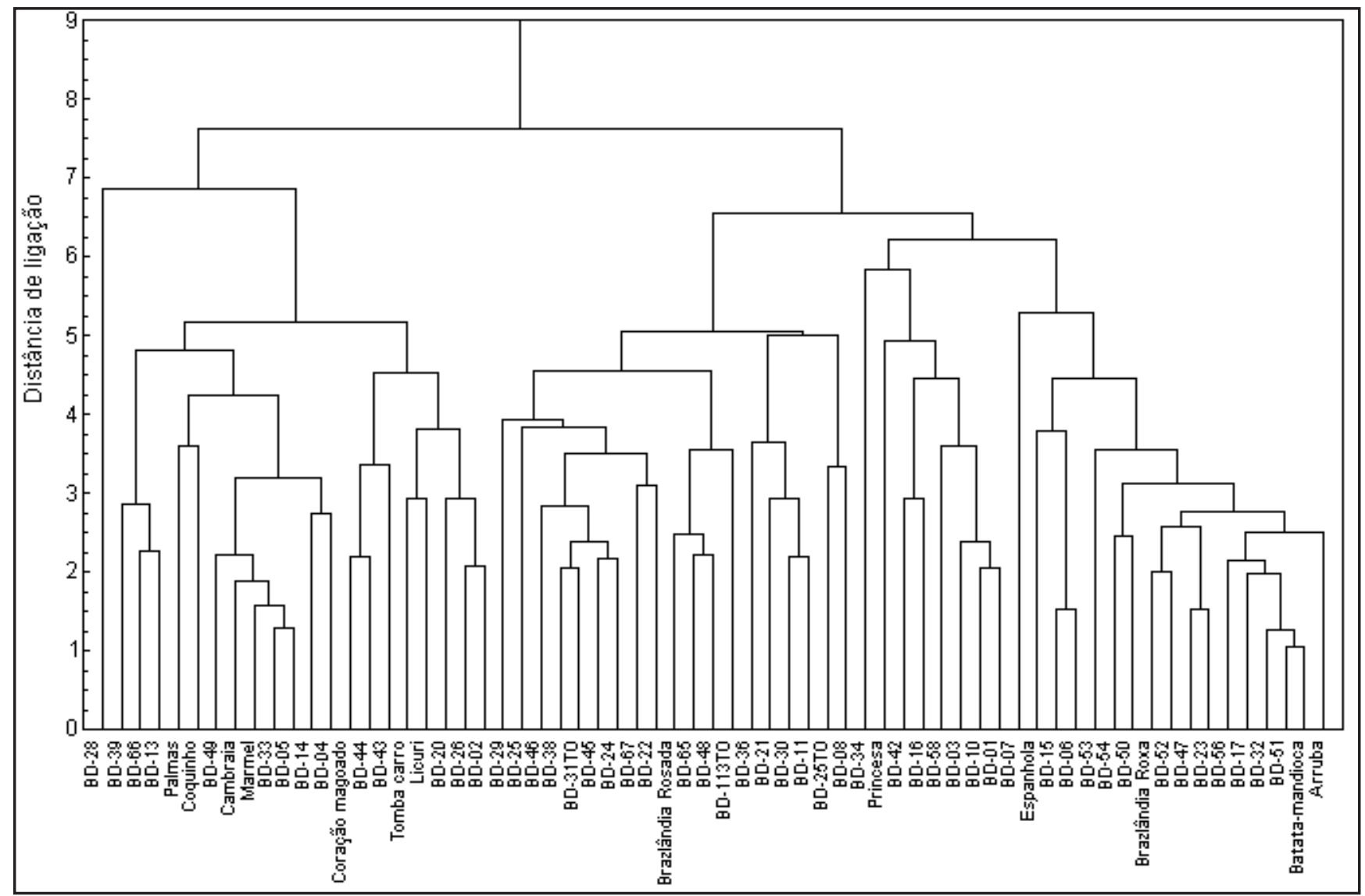

Figura 1. Dendrograma de dissimilaridade morfológica de 65 clones de batata-doce, estabelecido pelo método UPGA utilizando-se a distância Euclidiana, com base em 13 caracteres morfológicos (euclidian dissimilarity based on morphological traits of 65 sweet potato accessions and grouped according to the UPGA method). Diamantina, UFVJM, 2008.

BD-11, BD-25TO, BD-08, BD-34, Princesa, BD-42, BD-16, BD-58, BD03, BD-10, BD-01, BD-07, Espanhola, BD-15, BD-06, BD-53, BD-4, BD-50, Brazlândia Roxa, BD-52, BD-47, BD23, BD-56, BD-17, BD-32, BD-51,
Batata Mandioca e Arruba.

Com fundamento nestes resultados, pode-se confirmar a alta variabilidade morfológica entre os clones de batata-doce (Figura 1), com ausência de fenótipos iguais, reforçando a diversidade advinda dos parentais. Souza et al. (2006) caracterizando morfologicamente vinte acessos de batata-doce em Palmas-TO, observaram a formação de dois grandes grupos (90\% de divergência) com subdivisões. Ritschel \& Huamán 
(2001) avaliando 324 acessos nativos de batata-doce, coletados em todas regiões brasileiras, identificaram três grupos de acessos morfologicamente duplicados (GM1, GM2 e GM3).

Observa-se pelo dendrograma de dissimilaridade morfológica, que os clones mais similares morfologicamente são BD-51 e Batata Mandioca e os mais dissimilares são BD-28 e BD-07. Souza Júnior et al. (2003) avaliando características fisiológicas de cultivares de batata-doce, encontraram comportamento semelhante quanto à massa aérea nas cultivares Paraná, ESAM 2 e ESAM 3.

Verifica-se que, o perfil geral da folha (PG), número de lóbulos da folha (NL), pigmentação das nervuras inferiores (PNI), tipo de lóbulo da folha (TL) e a pubescência do ápice das ramas (PAR) foram às características que mais proporcionaram variabilidade entre os acessos.

\section{AGRADECIMENTOS}

Os autores agradecem à Fundação de Amparo à Pesquisa do Estado de Minas Gerais (FAPEMIG), processos CAGAPQ-7899-3.08/07 e EDT-407/07, Conselho Nacional de Desenvolvimento Científico e Tecnológico (CNPq), processo 566890/2008-9 e Coordenação de Aperfeiçoamento de Pessoal de Nível Superior (CAPES), processo PROCAD NF 2008/CAPES pelo auxílio financeiro e concessão de bolsas de estudos.

\section{REFERÊNCIAS}

ANDRADE JÚNIOR VC; VIANA DJS; FERNANDES JSC; FIGUEIREDO JA; NUNES UR; NEIVA IP. 2009. Selection of sweet potato clones for the region Alto Vale do Jequitinhonha. Horticultura Brasileira 27: 389-393.

AZEVEDO SM. 1995. Avaliação de famílias de meio-irmãos de batata-doce (Ipomoea batatas (L.) LAM.) quanto à resistência aos nematóides do gênero Meloidogyne e insetos de solo. Lavras: UFLA. 51 p (Tese mestrado).

BERNARDO S; SOARES AA; MANTOVANI EC. 2008. Manual de irrigação. Viçosa: UFV. $625 \mathrm{p}$.
BEUSELINCK PR; STEINER JJA. 1992. Proposed framework for identifying, quantifying and utilizing plant germplasm resources. Field Crops Research 29: 261-272.

BRITO JF; MOMENTE VG; SILVEIRA MA; SILVA LMA; NETO JJD; RIBEIRO PAA; BRITO DR; SANTOS GF. 2003. Avaliação de clones de batata-doce nas condições de Gurupi-TO. Campus universitário de GurupiTO da Universidade Federal do TocantinsUFT. Anais... Gurupi: UFT. 5 p.

CARDOSO AD; VIANA AES; RAMOS PAS; MATSUMOTO SN; AMARAL CLF; SEDIYAMA T; MORAIS OM. 2005. Avaliação de clones de batata-doce em Vitória da Conquista. Horticultura Brasileira 23: 911-914.

CRUZ CD. 1997. Programa Genes: aplicativo computacional em genética e estatística. Viçosa, MG: UFV. 442p.

DAROS M; AMARAL JÚNIOR AT. Adaptabilidade de produção de Ipomoea batatas. 2000. Acta Scientiarum.Agronomy 22: 911-917.

DAROS M; AMARAL JÚNIOR AT; PEREIRA TNS; LEAL NR; FREITAS SP; SEDIYAMA T. 2002. Caracterização morfológica de acessos de batata-doce. Horticultura Brasileira 20: 43-47.

DOMINGUES ET; SOUZA VC; SAKURAGUI CM. 1999. Caracterização morfológica de tangerinas do banco ativo de germoplasma de citros do centro de citricultura Sylvio Moreira/ IAC. Scientia Agricola 56: 197-206.

EMBRAPA-Centro Nacional de Pesquisa do Solo. 2007, 20 de setembro. Embrapa Solos. Rio de Janeiro. 12p. (Embrapa Solos. Documentos, 22). Disponível em: <http://www.cnps. embrapa.br/publicacoes $>$.

FAO. FOOD AND AGRICULTURE ORGANIZATION OF THE UNITED NATIONS. 2010, 02 de março. Sistema de dados agrícolas atualizados. Disponível em: $<\mathrm{http}$ ://faostat.fao.org/site.

FERREIRA DF. Sistema Sisvar para análises estatísticas. 2003. Versão online 5.0 (Build 67). Lavras, Universidade Federal de Lavras.

FERREIRA MAJF; QUEIROZ MA; BRAZ LT; VENCOVSKY R. 2003. Correlações genotípicas, fenotípicas e de ambiente entre dez caracteres de melancia e suas implicações para o melhoramento genético. Horticultura Brasileira 21: 438-442.

FIGUEIREDO JA. 2010. Seleção de clones de batata-doce com potencial de utilização na alimentação humana e animal. Diamantina: UFVJM. 54p (Dissertação mestrado).

FILGUEIRA FAR. 2008. Novo manual de olericultura. Viçosa: UFV. 421p.

FRANKEL OH; BENNET E. 1970. (ed.). Genetic resources in plants: their exploration and conservation. Oxford: Blackwell, 554 p.

HUAMÁN Z. 1996. Morphologic identification of duplicates in collections of Ipomoea batatas. Lima: International Potato Center. 28p. (CIP Research Guide, 36).

IBGE. INSTITUTO BRASILEIRO DE GEOGRAFIA E ESTATÍSTICA. 2010, 13 de janeiro. Sistema de informação. Disponível em: http://ibge.gov.br/estados/temas.

IBGE. INSTITUTO BRASILEIRO DE GEOGRAFIA E ESTATÍSTICA. 2009, 23 de dezembro. Sistema de informação. Disponível em: http://ibge.gov.br/estados/temas.

OLIVEIRA ACB; SEDIYAMA, MAN; SEDIYAMA T; CRUZ CD. 2000. Avaliação da divergência genética em batata-doce por procedimentos multivariados. Acta Scientiarum 22: 895-900.

RAMOS SRR; QUEIROZ MA. 1999. Caracterização morfológica: experiência do BAG de cucurbitáceas da Embrapa SemiÁrido, com acessos de abóbora e moranga. Horticultura brasileira 17, suplemento, p. 9-12.

RITSCHEL PS; HUAMÁN Z. 2001. Variabilidade morfológica da coleção de germoplasma de batata-doce da Embrapa-Centro Nacional de Pesquisa de Hortaliças. C. postal 218, 70359970, Brasília-DF.

SCAPIM CA; CARVALHO CGP; CRUZ CD. 1999. Uma proposta de classificação dos coeficientes de variação para a cultura do milho. Pesquisa Agropecuária Brasileira 30: 683-686.

SCOTT AJ; KNOTT MA. 1974. A cluster Pratissoli et al. Março, 1998 An. Soc. Entomol. Brasil 27: 115 analysis methods for grouping mean in the analysis of variance. Biometrics 30: $507-512$.

SOUZA AB. 2000. Avaliação de cultivares de batata-doce quanto a atributos agronômicos desejáveis. Ciênci. Agrotec. 24: 841-845.

SOUZA JÚNIOR AJL; MOREIRA JN; COSTA FB; SANTOS MA; NUNES GHS; SILVEIRA SF. 2003. Caracteres fisiológicos de cultivares de batata-doce: I - colheita aos quatro meses. Anais... ESAM-Departamento de Fitotecnia, Mossoró-RN.

SOUZA RC; OLIVEIRA JÚNIOR WP; CHIESA VB; TAVARES IB; SILVEIRA LR; ROSA W S; MOMENTE VG; SILVEIRA MA. 2006. Caracterização morfológica de vinte acessos de batata-doce provenientes do banco de germoplasma da UFT, Palmas-TO. Anais... Palmas: UFT. p.1633-1636.

STATSOFT INC. 2000. Statistica for Windows (Computer Program Manual). Tulsa: Statsoft, Inc.

STRAUSS MS; PINO JA; COHEN JI. 1989. Quantification of diversity in ex-situ plant collections. Diversity 16: 30-32.

VIANA DJS. 2009. Produção e qualidade de raizes, ramas e silagem de ramas de clones de batata-doce em diferentes locais e épocas de colheita. Diamantina: UFVJM. 69p (Dissertação mestrado). 